\title{
On Our Multi-Wavelength Campaign of the 2011 Outburst of T Pyx $\dagger$
}

\author{
L. Schmidtobreick, A. Bayo, Y. Momany, V. Ivanov, D. Barria, \\ Y. Beletsky, H. M. J. Boffin, G. Brammer, G. Carraro, W.-J. de Wit, \\ J. Girard , G. Hau, M. Moerchen, D. Nuernberger, M. Pretorius, \\ T. Rivinius, R. Sanchez-Janssen, F. Selman, S. Stefl, and I. Yegorova \\ European Southern Observatory, Casilla 19001, Santiago 19, Chile \\ email: 1schmidt@eso.org
}

\begin{abstract}
The well-known recurrent nova T Pyx has brightened by 7 magnitudes, starting on 2011 April 14, its first eruption since 1966. T Pyx is unique amongst recurrent novæ in being surrounded by a nebula formed of material ejected during previous eruptions. The latest eruption therefore offers the rare opportunity to observe a light echo sweeping through the existing shell, and a new one forming. The sudden exposure of the existing shell to high-energy light is expected to result in a change of the dust morphology as well as in the part destruction of molecules. We observe this process in the near- and mid-IR during several epochs using ESO's VLT instruments SINFONI, VISIR and IsAAC. Unfortunately, in the data analysed so far we only have a tentative detection in $\operatorname{Br} \alpha$ from the shell, so might in the end have to be content with upper limits for the emission from the various molecular bands and ionised lines.
\end{abstract}

Keywords. novae, ISM: molecules, dust

\section{Introduction}

Recurrent novæ $(\mathrm{RNe})$ are a class of cataclysmic variables that erupt at intervals of several decades. The eruptions are caused by a thermonuclear runaway (TNR) on the surface of a white dwarf accreting hydrogen-rich matter from a close companion star. The same mechanism is thought to be responsible for classical nova eruptions, whose recurrence times are, however, of the order of thousands of years instead of decades (Patterson 1984; Shara et al. 1986).

T Pyx - a recurrent nova - had five previous recorded eruptions (in 1890, 1902, 1920, 1944, 1966), and started the sixth on 2011 April 14. The accretion rate of the white dwarf is at least 100 times higher than expected for its orbital period (Uthas et al. 2010) and it is suggested that it has a white-dwarf mass close to the Chandrasekhar limit, thus making it a particularly good candidate as a type Ia SN progenitor (see also Knigge et al. 2000). It is surrounded by at least five dust and gas shells that were expelled during previous eruptions. Part of the nova remnant has been imaged both from the ground (where it appears as a roughly spherical shell) and with HST, where it is resolved into many individual knots (Shara et al. 1997; Schaefer et al. 2010).

\section{Data}

Although the shell is mostly invisible in the optical and near-IR continuum, we expect to find emission in certain frequencies in the IR centred on ionised lines. To our knowledge, none of the known nova shells has ever been observed in the mid-IR, but reference data

$\dagger$ Based on data of the UT3/UT4-team observatory project with program ID 287.D-5021. 
from similar objects like SN 1987A and V838 Mon indicate the formation of molecules and dust. Furthermore, the formation of dust has been observed during nova outbursts themselves: see, e.g., Nielbock \& Schmidtobreick (2003) for the outburst of V4745 Sgr. As the ionisation front from the current eruption sweeps through the shell we expect it to brighten in the UV and optical regions, amd in the Balmer lines and lines of ionised metals. The evolution of the shell is being monitored with the ESO's VLT instruments Visir, Sinfoni and IsAaC.

\subsection{VISIR}

Imaging for our first epoch was carried out in early May in PAH1, PAH2, Ne II, Ar III and S IV filters. The central source (T Pyx) was clearly visible in all regions. Several faint features are present in the surroundings but a careful analysis showed them all to be detector artifacts. So far, no detection of the shell itself can be claimed in any of the observations.

\subsection{SINFONI}

SinfONI integral-field spectroscopy was carried out at two epochs, the first directly after the nova outburst and the second about two months later. Even after a careful reduction of the data and a thorough search through the spectra we did not find any emission that could be attributed unambiguously to a shell around T Pyx. The images are instead dominated by stray light and remnants of the central source reaching out as far as $7^{\prime \prime}$, and thus make a clear distinction of the shell impossible.

\subsection{IsAAC}

IsAAC LW imaging was carried out with two narrow-band filters centred on $4.07 \mu \mathrm{m}(\mathrm{Br} \alpha)$ and $3.80 \mu \mathrm{m}\left(\mathrm{H}_{2}\right)$, and with the broad-band $L$ filter. So far only one epoch (directly after the nova explosion) has been reduced and analysed. The central source is present in all three filters. We have a tentative detection of the brighter parts of the shell in Br $\alpha$. The shell was not detected in the $L$-band continuum or in molecular hydrogen.

\section{Conclusion}

It appears that the old shell is still very weak in the early epochs after the nova outburst. That is to be expected, since the ionising light of the new explosion has not yet reached the old shell. Depending on which distance is adopted for T Pyx, the light will take between 8 and 20 weeks to reach the ring of material at a distance of about $5^{\prime \prime}$. The data taken at later epochs have not yet been analysed, but we are working on them and will discuss the new information together with the non-detections at early epochs in a forthcoming paper.

\section{References}

Knigge, C., King, A. R., \& Patterson, J. 2000, A\&A, 364, 75

Nielbock, M. \& Schmidtobreick, L. 2003, A\&A, 400, 5

Patterson, J. 1984, ApJS, 54, 533

Schaefer, B., Pagnotta, A., \& Shara, M. 2010, ApJ, 708, 381

Shara, M., Zurek, D. R., \& Williams, R. E., et al. 1997, ApJ, 114, 258

Shara, M., Livio, M., Moffat, A. F. J., \& Orio, M. 1986, ApJ 311, 163

Uthas, H., Knigge, C., \& Steeghs, D. 2010, MNRAS 409, 237 